\title{
REASONS OF USING ARABIZI AMONG \\ ENGLISH LANGUAGE STUDENTS IN JORDAN
}

\author{
Mahmoud Radwan Bardaweel ${ }^{(1)}$ \& Luqman M Rababah ${ }^{(2)}$ \\ School of Arts and Languages, Jadara University, Irbid, Jordan ${ }^{(1)}$ \\ Associate Professor - School of Arts, Jadara University, Jordan ${ }^{(2)}$ \\ luqman@jadara.edu.jo
}

\begin{abstract}
The purpose of this study is to investigate the reasons for utilizing Arabizi among students at Jadara and Yarmouk Universities, as well as the prevalent motivations for switching for each reason. For the academic year 2019/2020, this quantitative study includes students studying English language and translation at two institutions, Yarmouk and Jadara. The participants in this study were picked at random from Jadara and Yarmouk Universities in the Irbid governorate. This study's data comes from a questionnaire that was given to 283 students of both genders (male and female). The researchers used Standard Deviations, percentages, means, frequencies, statistical figures, and the Friedman test to answer the study's research question. The findings revealed that there are numerous explanations for this. It is used by the participants as a prestigious language, as well as an easy way to write and appear more westernized. Code switching has three motivations: communication, intellectual, and solidarity. Because this study was limited to the governorate of Irbid, which is located in northern Jordan, there is a need to investigate the reasons in other Jordanian geographic locations.
\end{abstract}

Keywords: Arabizi, Code Switching, Sociolinguistics, Reasons. 


\section{Introduction}

Modern technologies are affecting all ways of communication, and due to the modernization and globalization we have been witnessing recently, a new way of communication has appeared to dominate our daily life interactions known as Arabizi the process of replacing Arabic letters with Latin letters and numerals. One purpose of Arabizi is that it shortens the phrases or the words of Arabic language. People tend to use Arabizi more in their daily social interactions due to many different reasons in our society. Arabizi simply facilitates the process of pronouncing Arabic letters if the speaker is intending to pronounce them in English such as the Arabic letter ( $ح$ ) (haah) can be written as (7). Example; B7ibak = I love you. (Saide, 2019; Al-Khatib \& Sabbah 2008).

Some reasons behind using Arabizi by some Arab youths are because it is considered a coding communicative language among them, and because it is somehow more expressive than Arabic language. It is also revealed that this new language is widely used by individuals and youths of same group age and not used with elder people or parents, also it is not used in formal writing. Furthermore, Arabizi dominantly used in social writing and chatting and not used in Academic, religious, literature, scientific, or business topics (Alsulami, 2019).

It is indicated in the same line of the research that there have been few studies conducted on the scope of Arabic - English code mixing or what is known as Arabizi, Arabish, Franco-Arabic, or Arabiglish (Alghamdi \& Petraki, 2018; Al-Shaer 2016). It is basically used by bilingual or multilingual society. It was first used by Arab expatriates whether on the internet or by using the cellular phones when the actual Arabic letters weren't existed or unavailable because of technical issues, so Arabizi was used at that time to replace the original Arabic. Due to the globalization we have been witnessing since the last decade of the last century especially in various aspects of various media platforms youths started to think that Arabic language doesn't enhance globalization and they insisted on using Arabizi to go with the modernization; furthermore, lots of social media platforms and many various web pages appeared and developed to help using Arabizi. (Kenali, Yusoff, Kenali, \& Kamarudin 2016; \&Ash-Shammari \& Al-Enzi 2012).

The studies made by (A1-Shaer 2016; Darwish 2013 and Al-Khatib \& Sabbah 2008) regarding the reasons of using Arabizi phenomenon in our daily social interactions are varied and different; some of them found out that people tend to use Arabizi for show off and some said that people use Arabizi because it is considered as a prestigious language. In spite of the fact that there have been lots of studies carried out on various aspects of the sociolinguistics perspective regarding Arabizi, few studies were conducted on gender 
differences in using Arabizi especially on social networking platforms (Alghamdi \& Petraki, 2018). Moreover, few studies have discussed the use of it among various English language students particularly students of Jadara and Yarmouk Universities.

Significance of the study

This study is unique because it focuses on the reasons behind using Arabizi, the common motivations for code switching, based on certain variables such as age, and place of living. Moreover, by using the quantitative methodological approach, the present study contributes to methodology in terms of the approach that it makes use of. Furthermore, by examining the reasons and a sociolinguistic perspective of gender differences, this study bridges a gap in the previous literature.

Since there were minimal studies existing in the past literature to convey the improvements and the modernization, this study is important because it handles this phenomenon more comprehensively, tries to target a larger number of university students, and obtain more data; it also attempts to include more statistical data about which gender tend to use this phenom-

\begin{tabular}{|c|c|c|c|c|}
\hline (E.g.) & Numeral & Equivalent in English & Name of the Letter & $\begin{array}{l}\text { Arabic } \\
\text { letter }\end{array}$ \\
\hline $\begin{array}{l}\text { Ana; }\{\text { ana }\} \\
(\mathrm{I} / \mathrm{me})\end{array}$ & 2 & $\bar{A}$ & Hamzah wa Alif & $\begin{array}{l}i_{\varphi} \\
\end{array}$ \\
\hline $\begin{array}{l}3 \text { my ; \{'amy\} } \\
\text { (my Uncle ) }\end{array}$ & 3 & 'a or 'e & 'Ayn & $\varepsilon$ \\
\hline $\begin{array}{l}\text { 3'yab; \{ghiab }\} \\
\text { (Absence ) }\end{array}$ & $3^{\prime}$ & gh & Ghayn & $\dot{\varepsilon}$ \\
\hline $\begin{array}{l}\text { 5atim; }\{\text { Khatim }\} \\
\text { (Ring) }\end{array}$ & 5 & $\mathrm{kh}$ & Khaa & $\dot{\tau}$ \\
\hline $\begin{array}{l}\text { 6abeeb; }\{\text { Tabeeb }\} \\
\text { ( Doctor) }\end{array}$ & 6 & $t($ Sharp t ) & Taa & b \\
\hline $\begin{array}{l}7 \mathrm{ob} ;\{\text { hob }\} \\
\text { (Love ) }\end{array}$ & 7 & h ( sharp h) & Haа & $\tau$ \\
\hline $\begin{array}{l}\text { Da8ee8a; }\{\text { daqeeqa }\} \\
\text { (Minute) }\end{array}$ & 8 & Q & Qaaf & ق \\
\hline $\begin{array}{l}\text { 9ba7 el5air; } \\
\text { \{sabah al khair } \\
\text { (Good Morning) }\end{array}$ & 9 & s ( sharp s ) & Saad & ص ص اص \\
\hline $\begin{array}{l}21 \text { 4al4; \{al thalith\} } \\
\text { ( The Third ) }\end{array}$ & 4 & th & Thah & $ث$ \\
\hline $\begin{array}{l}\text { 9'ab6 ; }\{\text { Dhabit }\} \\
\text { ( Officer ) }\end{array}$ & $9^{\prime}$ & $\mathrm{dh}$ & Dhad & ض \\
\hline
\end{tabular}


enon. Moreover, this study is to answer the calls made by (Alghamdi \& Petraki, 2018; Al-Shaer 2016; Darwish 2013 and Al-Khatib, $\&$ Sabbah 2008) to investigate the reasons.

\section{Literature review}

\section{Arabizi}

Al-Bayati (2018), stated that Arabizi has no systematic form of writing and there is no fixed way to write it because there are many different dialects and accents. Basically Arabizi is based on transliteration of Arabic text written using numbers and Latin characters that represent some letters in Arabic for example using English to write Arabic and that the letters which are not exist in English or have no equivalent can be represented by using the Roman numerals; e.g the letter $ح$ )) has

Tabel 2.2 some expressions used in arabizi

\begin{tabular}{|c|c|c|}
\hline Abbreviation & Meaning in English & Meaning in Arabic \\
\hline FYI & For your information & المعلوماتكا \\
\hline OMG & Oh my God & يا ل يالهي \\
\hline BRB & Be Right Back & ساعود لاحقا \\
\hline LOL & Laughing out loud & ضحك بصوت عالي \\
\hline IDC & I don't care & لا اهتم \\
\hline BTW & By the way & بالمناسبة \\
\hline TTYL & Talk to You Latter & بكلمك بعدين \\
\hline
\end{tabular}

no equivalent in English so that it can be represented in Arabizi by numeral the (7). Here is a list of the common numerals and the letter they stand for (see table 2.1).

Table 2.1 List of common numerals used in Arabizi

Izzeldein (2017) defines Arabizi as a process of using Latin letters and numbers instead of Arabic letters, and it is another model language used in modern technology; this phenomenon ultimately led to the invention of new shortcuts of certain expressions spread among the youths and they are certain letters carry a bigger meaning and expressions inside (see table 2.2).
Tobaili (2016) sought in his study to define Arabizi as a digital trend using Latin scripts in texting nonstandard Arabic, and its users don't use unified orthography. The results of the study show that the percentage of Arabizi usage across Lebanon and Egypt in mobile text messaging is higher than it is in Twitter medium. he hypothesizes that this is because people prefer using it on private medium than the public medium because this way of communication perceived as informal communication way. Another aspect of the research reveals that there are some tools available such as Microsoft Maren, Yamli, and Google input tools help transliteration into Arabic; these tools function as translators from infor- 
REASONS OF USING ARABIZI AMONG

ENGLISH LANGUAGE STUDENTS IN JORDAN

mal language which is Arabizi into standard Arabic.

\section{Code switching/ mixing}

According to Poplack (2001),code-switching is the process of mixing two or more languages by bilingualism or multilingualism in their daily speaking, normally with no change to the topic and it may happen at any level of a linguistic structure, but it occurs within the limit if a single sentence constituent, or a single word. Even though it is being used widely nowadays by different people worldwide, it started to attract scholars and researchers attention.

As to Myers-Scotton (1995), Code-Switching is a variety of language in which the speaker whether bilingual or multilingual alternates between two or more linguistic varieties in the context of conversation; the varieties participating in code switching may be dialects, different languages, or styles. Scotton adds that Code-Switching is considered as a type of a skilled performance of a speaker who conveys the process of alternating between two or more linguistic varieties. And reasons for doing so are because showing solidarity, eloquence, distance, or difference.

As to Eldin (2014) \&Al-Khawaldeh, Al-Khawaldeh, Bani-Khair,\& Algwery (2016), code switching is used commonly among Facebook users and different media mediums because it is considered a tool that prompts and supports their interactions in Facebook and the other media mediums. It is also con-
Jadara Journal for Studies and Research

Volume (seventh) for the year (2021)

sidered a strategic tool by means of which meanings are effectively communicated and delivered. The process of manipulation both languages English and Arabic helps the users achieve well both their stylistic and communicative purposes. It is needed from those who use switching to be fully competent and both languages and to note that code switching phenomenon occurs not only in speech converse but rather in electronical in written discourse. It is proven in this study that the need for facilities, lack of efficiency, and common habitual expressions are the main causes for switching. Along with that the speaker's mood is a main contributing reason for switching also.

According to (Muthusamy, 2009; Holms, 2013; poplack 2013; Wei, 2000; and Myers-Scotton 1995) there are various motives for Switching and the most common ones are: First motive for switching is Communicative motive: This motive for switching takes place in order to ease the communication process by using short and easy routes, for more effective communication, to demonstrate support and good will, for the purpose of establishing certain effects of communications, and because the speaker is intending to exclude some people from a dialogue.

Second motive is conceptual motive: This motive for switching takes place because of semantic reasons, habitual facts, to clarify a misunderstanding, and because some experiences were experienced in a certain language. Third motive for switching is Social distance 
motive: This motive for switching typically takes place between the participants and they motivated by their identity or relationship. Third motive of Switching known as Lexical motive: This motive for switching normally takes place in order to express precise meanings, for effective communication, a need for facility, and there is a shortage of certain vocabularies in a language; when speaking a new language speakers may use original words from their mother tongue to convey the missing one, fourth motive for Switching which is group identity: This motive for switching serves as ethnic identity maker to show solidarity.Fifth, there is as well a motive for Switching known as effective Function motive: This motive for switching takes place when somebody is looking for effective function rather than referential meaning. The sixth motive for Switching is psychological motive: This motive for switching basically based on the mood of the speaker.

The seventh motive for switching is interlocution motive: This motive for switching typically takes place in order to invoke the listeners, to fill a gap, to express solidarity, to speak to a different audience, and to show a group identity. The Eighth motive for switching is emphasis motive: This motive for switching typically takes place in order to attract the attention of the hearers, to confirm a point, and when the speaker wants to add or make a point.

According to (Deuchar, 2012; and Holms, 2013) there is another motive for Switching known as Technical Switching: This motive for switching typically takes place due to technical difficulties of certain types of communication's devices.

Finally according to (Maros, Noorizan, \& Abd Akim, 2016; and Holmes, 2013) there is another motive for Switching which is Solidarity motive: this motive for switching occurs when the hearer respond with the same code switching then the relation between both will be established; and because the speaker feels the need to belong to another group or identity.

\section{Related studies}

Al-Khatib and Sabbah (2008) conducted a sociolinguistic study on 46 Jordanian university students. The study shows that $61 \%$ tend to use Arabizi in their daily social contact: $39 \%$ used pure Arabizi, $54 \%$ used English words and phrases, and just $7 \%$ used Arabic in their social contact. The study also shows that contrary to males women tend to use Arabizi more $44 \%$ of females tend to use Arabizi in their daily social contact for the sake of showing off, gaining prestige or employing euphemism. The study also discloses the choice of the language variety (i.e. Arabizi, English, or Arabic) for an extant depends on the topic. For example, Arabizi, which is widely used among the university students was mostly employed in intimate social and personal interactions. While Arabic was used in topic related to religious and cultural areas; on the other hand English was used in the 
topic attached to work or university matters.

In a similar study conducted by Al-Shaer (2016).The study shows that using Arabizi was linked with weaker scores on the spelling test, showing that the use of Arabizi harms students Arabic capabilities; moreover, there are many other factors prompts the students to use Arabizi which results in having poor Arabic spelling skills.Additionally, Darwish's (2013) study showed that Arabizi is used widely nowadays in informal social media platforms and it is used in order to show both modern standard Arabic, The researcher introduces many other names for Arabizi such as Arabish, Franco-Arab, and many other names.

Hamdan (2017) indicates in the same line of the research that Face to face interaction began to be shifted to Computer Mediated Communication (CMC) with the growth of communication and the internet. Basically there are many variables play crucial roles in determining the use and non-use of Arabic Romanization. One reason for that is the students major, it is presented in this study that the student's major play an important role in the language shift; it is also proven that the student's major contributes to shape their attitude toward this kind of writing e.g. it is stated that the students who study English and students of medicine who receives their education in English were more familiar with Arabic Romanization than the students of Arabic and the students of Sharia who receives their education in Arabic.
Hamdan (2017) added that it is stated from users and non-users of the Romanized language there will be a danger in the long run on Arabic language if people continue to use this phenomenon at the current rate. Additionally he added that there are various reasons for using this kind of Romanization and the most common ones are it made the process of typing Latin letters easy and swift, and it helps it's users to get accommodated with other partner with whom he is interacting.

Alghamdi \& Petraki (2018) conducted a study in which they found out the reasons why young Saudi Arabians tend to use this phenomenon online are : 1) their peers language 2 ) considered a stylish language 3 ) they face difficulties with their original Arabic Language 4) it is a secret code. The study concluded that group solidarity and Arab youth identity are marked strongly by Arabizi. One aim of this study is to give the Saudi Arabian youths a voice to express their attitudes and opinions regarding this phenomenon without any negative imposition. It is demonstrated in this study that young Saudis and their peers are using Arabizi as a kind of a unique and a redefined identity for them. Eventually by using Arabizi they are showing the world that they have a different and a creative style. Furthermore, it is recommended in this study to investigate the potential sex differences between both gender (Males\& Females ) regarding the use of Arabizi and their attitude as well. Adding that no gender differences were reported in this study with respect to 
certain variables such as Socioeconomics, level of education, or institution of education.

After reviewing the previous studies, one can observe that few studies have examined the reasons behind using Arabizi internationally and locally (Alghamdi \& Petraki, 2018\&Hamdan , 2017). Thus, this study bridges a gap in the previous literature.

\section{Methods}

This study is in quantitative method approach. The population of this study contained the students who study English language and translation in two universities namely Yarmouk and Jadara for the Academic year 2019/2020. This study has been conducted in both English literature and translation departments at Jadara and Yarmouk Universities. A permission has been gained from both universities to allow the researcher to enter both departments and disseminate the questionnaire. The sample of this study contained 283 participants chosen randomly from nearly males and females students who study at Jadara and Yarmouk Universities locate at Irbid governorate. The data in this study involves a questionnaire which was distributed to 283 students from both genders (male and female). To answer the first question of the study the researcher used the Standard deviations, percentages, means, frequencies, statistical figures, and Friedman test.

\section{Results and discussion}

In order to achieve the aims of the study, a questionnaire was utilized on a convenience sample comprising fifty students and ten EFL teachers in DSs. The present section presents the findings based on the tool used to answer the following research questions:

1. What are the reasons behind using Arabizi among English language students at Jadara and Yarmouk universities?

2. What are the motivations of code switching for each reason?

1 The first research question: What are the reasons behind using Arabizi among English language students at Jadara and Yarmouk universities?

To answer the first research question entitled, the researcher has used percentages, means, statistical figures, and Friedman test as shown in tables 4.1. The first question is answered based on relative importance (Friedman test) where the mean rank, and percentages show the most common reasons for using Arabizi and they are arranged from the common use to least use as concluded from the results of the questionnaire. 
Table 4.1 The results of Friedman test for the most common reasons of using Arabizi

\begin{tabular}{|c|c|c|c|c|}
\hline $\begin{array}{l}\text { The reasons behind using Arabizi among Jordanian } \\
\text { Iniversity students( Jadara and Yarmouk universities) }\end{array}$ & Mean Rank & $\% 100$ & $\begin{array}{l}\text { FriedmaN } \\
\text { value }\end{array}$ & p.value \\
\hline $\begin{array}{l}\text { use Arabizi because it is considered as a prestigious } \\
\text { anguage. }\end{array}$ & 9.12 & $8.5 \%$ & 62.332 & \multirow{14}{*}{$\begin{array}{l}\text { Less } \\
\text { than } \\
0.01\end{array}$} \\
\hline I use Arabizi to appear more westernized. & 8.82 & $8.4 \%$ & & \\
\hline use Arabizi because it is easier than writing pure Arabic. & 8.06 & $7.7 \%$ & & \\
\hline $\begin{array}{l}\text { use Arabizi because it is considered nowadays as a life- } \\
\text {;tyle. }\end{array}$ & 7.91 & $7.5 \%$ & & \\
\hline use Arabizi because of privacy issues. & 7.86 & $7.5 \%$ & & \\
\hline 3ecause it is used commonly by my colleagues & 7.75 & $7.4 \%$ & & \\
\hline Need to cope with the new generation. & 7.43 & $7.1 \%$ & & \\
\hline Гo enhance my typing skills on English Language. & 7.34 & $7.0 \%$ & & \\
\hline use Arabizi because of personal interests. & 7.27 & $6.9 \%$ & & \\
\hline use Arabizi because of technical issues & 7.22 & $6.9 \%$ & & \\
\hline use Arabizi for the sake of showing off. & 7.02 & $6.7 \%$ & & \\
\hline use Arabizi because I don't like Arabic language. & 6.89 & $6.6 \%$ & & \\
\hline use Arabizi because of poor knowledge in Arabic. & 6.81 & $6.5 \%$ & & \\
\hline $\begin{array}{l}\text { use Arabizi to save money and time in the message that I } \\
\text { im writing because it spares more spaces. }\end{array}$ & 5.41 & $5.4 \%$ & & \\
\hline
\end{tabular}

Table 4.1 indicates that the sentence "I use Arabizi because it is considered as a prestigious language" is the most important reason from the point of view of the participants, where it's mean rank reaches to 9.12; and it's percentage reaches $8.5 \%$. It is also indicated that the sentence "I use Arabizi to save money and time in the message that I am writing because it spares more spaces" is the least important reason from point of view of the sample study, where it's mean rank reaches to 5.41; and it's percentage reaches $5.4 \%$. According to Sert (2005) speakers sometimes switch to influence, manipulate, or even to clarify a situation as they need, and possibly they do this to express themselves or may be to identify the language because of personal intentions.

Tabel 4.2 frequency and Percentages of the motivations for CS

\begin{tabular}{ccc}
\hline Motive for swiching & Frequency & Percentage \\
\hline Conceptual motive & 68 & 24 \\
Solidarity motive & 42 & 14.9 \\
Psychological motive & 30 & 10.6 \\
Interlocution & 20 & 7 \\
Lexical motive & 14 & 4.9 \\
Technical motive & 13 & 4.6 \\
Effective Function & 4 & 1.5 \\
Total & 283 & 100 \\
\hline
\end{tabular}


2 The second research question: What are the motivations of code switching for each reason?

To answer the second question entitled, the researcher has used percentages, and frequencies as shown in tables 4.2.Moreover; the researcher has made use of the questionnaire to answer the question using the frequencies and the percentages of each motive for code switching.

Table 4.2 Frequency and Percentages of the motivations for $\mathrm{CS}$

Total $283 \quad 100$ The researcher has made use of a questionnaire in order to answer the second question based on the frequencies and the percentages of the motivations for code switching. The obtained responses are divided into eight motivations for switching as shown in table 4.2 .

As table 4.2 shows that communicative motive has got the highest percentage among all motives for switching from the view of the participants. Ninety two participants have stated that they switch to enhance their typing skills on English Language, because of personal interests, because Arabizi is easier than writing pure Arabic, and because it is used commonly by their colleagues. This is because they are looking for more effective communication way, and they find it an easy way of communication because they can use short and easy routs. This constitutes 32.5 percent of the frequency ratio. This result is consistent with what has been stated in
(Muthusamy, 2009; Al-Shaer 2016; Darwish 2013 \& Al-Khatib \& Sabbah 2008) regarding the common motives and reasons for switching.

Additionally, as shown in table 4.2 conceptual motive has the second highest frequency and percentage among all motives for Switching from the view of the participants. Sixty-eight participants have stated that they switch because Arabizi is considered as a prestigious language, because it is considered nowadays as a life-style, and for the sake of showing off. This is because a need clarify themselves, because of their habit to use another language, and because of semantic significance. This constitutes 24 percent of the frequency ratio. This result supports what has been stated in (Muthusamy, 2009; Al-Shaer 2016; Darwish 2013 \& Al-Khatib \& Sabbah 2008) regarding the common motives and reasons for switching.

Moreover; it can be also noticed from table 4.2 that solidarity motive has got the third highest frequency and percentage among all motives of Switching from the view of the participants. Forty- two participants have stated that they switch to appear more westernized, and to cope with the new generations. This is because they feel the need to belong to group or identity, or because they want to respond with the same code switching. This constitutes 14.9 percent of the frequency ratio. This result is in line with what has been stated in (Muthusamy, 2009; Holms, 2013; Poplack 2013; Wei, 2000; Myers-Scot- 
REASONS OF USING ARABIZI AMONG ENGLISH LANGUAGE STUDENTS IN JORDAN

ton, 1995;\& Maros, Noorizan, \& Abd Akim, 2016) regarding the common motives and reasons for switching.

In addition, table 4.2 shows that psychological motive has the fourth highest frequency and percentage among all motives of Switching from the view of the participants. thirty participants have stated that they switch because of privacy. This is related to the mood of the speaker. This constitutes 10.6 percent of the frequency ratio. This result is consistent with what has been stated in (Muthusamy, 2009; Holmes, 2013; Poplack 2013; Wei, 2000; and Myers-Scotton 1995) regarding the common motives and reasons for switching.

Furthermore, it can also be noticed from table 4.2 that Interlocution motive has the fifth highest frequency and percentage among all motives of Switching from the view of the participants. Twenty participants have stated that they switch because they do not like Arabic language. This is because they want to provoke the listeners or to fill a gap. This constitutes 7 percent of the frequency ratio. This result supports what has been stated in (Muthusamy, 2009; Holmes, 2013; Poplack 2013; Wei, 2000; and Myers-Scotton 1995) regarding the common motives and reasons for switching.

As well, it can be also seen from table 4.2 that lexical motive has the sixth degree percentage among all motives of switching from the view of the participants. Fourteen participants have stated that they switch because of
Jadara Journal for Studies and Research Volume (seventh) for the year (2021)

poor knowledge in Arabic. This constitutes 4.9 percent of the frequency ratio. This is because they want to express a precise meaning, or to convey missing meaning from their mother tongue. This result is in line with what has been stated in (Muthusamy, 2009; Holms, 2013; poplack 2013; Wei, 2000; and Myers-Scotton 1995) regarding the common motives and reasons for switching.

Likewise, technical motive has got the sixth degree percentage among all reasons of Switching from the view of the participants. Thirteen participants have stated that they switch because of technical issues. This constitutes 4.6 percent of the frequency ratio. This result supports what has been stated in (Al-Khatib \& Sabbah 2008) regarding the common reasons for using Arabizi because this study had been carried out on past generations of communication devises where it is said that people are using Arabizi frequently in order to spare money and recently we have various aspects of free-cost social media platforms that don't need much expense.

Finally, table 4.2 shows that effective function switching has the least degree percentage among all motives from the view of the participants. Four participants have stated that they switch to save money and time in the message that they are writing, and because it spares more spaces. This constitutes 1.5 percent of the frequency ratio. This result is inconsistent with what has been stated in (Al-Khatib \& Sabbah 2008) regarding the common reasons for using Arabizi because 
their study had been carried out on past generations of communication devises in which it has been stated that people are using Arabizi frequently in order to save money, and recently we have new generations of various aspects of free-cost social media platforms that don't need much expense.

\section{Conclusion \& Recommendations}

The study aims to allocate and identify the reasons that prompts university students to use this newly appeared phenomenon and the common motivations for code switching. It was found in the study that Yarmouk and Jadara English students are using Arabizi according to the following reasons and they are arranged according to the most used and common causes: They use Arabizi because it is considered as a prestigious language, they use Arabizi to appear more westernized, they use Arabizi because it is easier than writing pure Arabic, they use Arabizi because it is considered nowadays as a life-style, they use Arabizi because of privacy issues, They use Arabizi because it is used commonly by my colleagues, they use Arabizi because there is a need to cope with the new generation, they use Arabizi to enhance my typing skills on English Language, they use Arabizi because of personal interests, they use Arabizi because of technical issues, they use Arabizi for the sake of showing off, they use Arabizi because I don't like Arabic language. They use Arabizi because of poor knowledge in Arabic, and they use Arabizi to save money and time in the message that I am writing because it spares more spaces. Furthermore, the study also revealed that there are various motivations for Code Switching and some of these motives are: Communication, Conceptual motive, Solidarity motive, Psychological motive, Interlocution, Lexical motive, Technical motive, and Effective Function motive. The study recommends examining other students who study different majors in these two universities or in other universities located in/ out the geographic region.

\section{References}

Al Heeti, N., \& Al Abdely, A. A. (2016). Types and functions of code-switching in the English language used by Iraqi doctors in formal settings. International Journal of Advanced Research and Review, 1(8), 10-18. Al-Bayati, R. (2018). Arabglish: Writing Arabic in English. Eton Institute.

Alghamdi, H., \& Petraki, E. (2018).Arabizi in Saudi Arabia: A Deviant Form of Language or Simply a Form of Expression?. Social Sciences, 7(9), 155-165.

Al-Khatib, M., \& Sabbah, E. H. (2008). Language choice in mobile text messages among Jordanian university students. SKY Journal of Linguistics, 21(1), 37-65.

Al-Khawaldeh, M., Al-Khawaldeh, N., Bani-Khair, B.\& Algwery, H. (2016). Mechatronics Engineers' perception of Code mixing: Philadelphia University and Hashemite University as a case study. International Journal of Applied Linguistics \& English Litera- 
ture,5(7),110-117.

Almwajeh, M., \& Rababah, L. (2019). There is more to it than meets the eye: an intercultural study of religious speech acts between Jordanian and American students. International Journal of Linguistics, 11 (1), 34-45.

Al-Shaer, I. M. (2016).Does Arabizi Constitute a threat to Arabic?.Arab WorldEnglish Journal, 4(3), 18-30.

Alsulami, A. (2019). A Sociolinguistic Analysis of the Use of Arabizi in Social Media Among Saudi Arabians. International Journal of English Linguistics, 9(6).

Bani-Ismail, I. T. M. (2012). Arabizi has Used by Undergraduate Students in Some Jordanian Universities: A Sociolinguistic Study (Doctoral dissertation, Yarmouk University).

Banikalef, A., \& Rababah, L. (2018). Gender differences and emotional expressiveness on Facebook: An analysis of prosodic features among Jordanian Facebookers. Studies in Linguistics and Literature, 2(3), 180-184.

Creswell, J. W. (2014). Educational research: Planning, conducting, and evaluating quantitative Research(4th Ed.). Pearson Education Ltd, (pp. 174-203).

Crystal, D. (2001). Language and the Internet. Cambridge: Cambridge University Press.

Darwish, E. B. (2017). Factors influencing the uses, diglossia and attrition of Arabic language in social media: Arab youth case. Journal of Education and Social Sciences, 7(1),
Jadara Journal for Studies and Research Volume (seventh) for the year (2021)

$250-257$.

Darwish, K. (2013). Arabizi detection and conversion to Arabic.

Deuchar, M. (2012). Code switching. The encyclopedia of applied linguistics.

Eldin, A. (2014). Socio linguistic study of code switching of the Arabic language speakers on social networking. International journal of English linguistics, 4(6), 78.

Farrag, M. (2012).Arabizi:a writing variety worth learning? an exploratory study of the views of foreign learners of Arabic on Arabizi.

Ghanem, R., (2011). Arabizi is destroying the Arabic language. Arab world.

Ghazal, M. (2014). Arabizi Popularity Threatening Arabic Proficiency among Native Speakers, Experts Warn.

Hanaef A, \& Al-Enzi, A. (2012). Al-'Arabizi-Asbab Wa Hulul. Kuwait.

Holmes, J. (2013). An introduction to sociolinguistics. Routledge Taylor and Francis group

Hudson, R. A. (1996). Sociolinguistics. Cambridge university press.

Izzeldein, A. (2017). Your guide to the youths language on the internet. Raseef 22.

Jdaitawi, M., Ishak. N., Taamneh, M., Gharaibeh, M., \&Rababah, L. (2011). The Effectiveness of Emotional Intelligence Training Program on Social and Academic Adjustment 
among First Year University Students. International Journal of Business and Social Science, 2 (24). 251-258.

Kenali, A., Yusoff, N., Kenali, H., \& Kamarudin, M. (2016). Code-Mixing Consumptions among Arab Students. Creative Education, 7(07), 931.

Kusuma, L. B. (2014). A study of leetspeak among young Hispanic Americans in mocospace mobile social network. (Doctoral dissertation), Sanata Dharma University.

Maros, M., Noorizan, N. D. M. M., \& ABD AKIM, I. Z. (2016). Code switching as the medium of solidarity in 'Ola Bola'. Jurnal Komunikasi: Malaysian Journal of Communication, 32(2).

Milroy, J. (1995). One Speaker, Two languages: Cross-disciplinary Perspectives on Code-Switching. Cambridge University Press.

Muthusamy, P. (2009). Communicative functions and reasons for code switching: A Malaysian perspective. Language \& Society, 5, 1-16.

Myers-Scotton, C. (1995). Social motivations for codeswitching: Evidence from Africa.
Oxford University Press.

Poplack, S. (1980). Sometimes I will start a sentence in English y termino en espan ol" Toward a typology of code-switching. Linguistics 18, 581-616.

Poplack, S. (2013). Sometimes I will start a sentence in Spanish Y TERMINO EN ESPAÑOL: Toward a typology of code Switching. Linguistics, 51, (Jubilee),(11-15).

Poplack, S.(2001). Code-switching (linguistic). International encyclopedia of the social and behavioral sciences, 12, 2062-2065.

Rababah, I., Rababah, L. (2017). Investigating Arabic to Speakers of Other Languages (ASOL) Lecturers' Attitudes towards Utilizing Flipped Classroom Instruction (FCI): A Qualitative Study at Jordanian Public Universities. International Educational studies, 10 (7), 80-91. https://doi.org/10.5539/ies. v10n7p94

Rababah, L., \& Bani Melhem, N. (2015). Investigation into Strategies of Creativity in EFL Writing in Jordan. Journal of Literature, Languages and Linguistics, 5 (23), 14-25.

Saide Jr, A. A. (2019). Arabizi-Help or Harm? An Analysis of the Impacts of Arabizi-Threat 
or Benefit to the Written Arabic Language? (Doctoral dissertation), University of Dayton.

Sert, O. (2005). The Functions of Code-Switching in ELT Classrooms. Online Submission, 11(8).

Sullivan, N. (2017). Writing Arabizi: Orthographic Variation in Romanized Lebanese Arabic on Twitter (Doctoral dissertation).

Tobaili, T. (2016). Arabizi identification in twitter data. In Proceedings of the ACL 2016 Student Research Workshop (pp. 51-57).

Warschauer, M., Said, G. R. E., \& Zohry, A. G. (2002). Language choice online: Globalization and identity in Egypt. Journal of Computer-Mediated Communication, 7(4). 155-170.

Yaghan, M. A. (2008). Arabizi: A contemporary style of Arabic Slang. Design Issues, 24(2), $39-52$.

Zuhair, A. (2015). The Effect of Arabism of Romanic Alphabets on the Development of 9th Grade English as a Foreign Language Students' Writing Skills at Secondary School Level. Journal of Education and Practice, 6(36), 91-95. 